\title{
RESEARCH AND MODELLING INDUCED ENERGY DISTRIBUTION Processes in The Ground Wire Cable
}

\author{
Iosif Breido, Vladimir Kaverin \& Sholpan Yezhebayeva
}
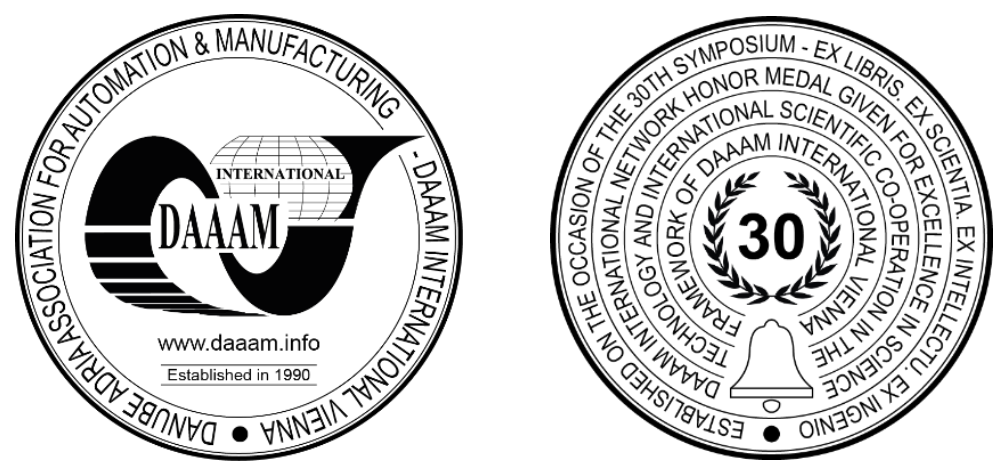

This Publication has to be referred as: Breido, I[osif]; Kaverin, V[ladimir] \& Yezhebayeva, S[holpan] (2021). Research and Modelling Induced Energy Distribution Processes in the Ground Wire Cable, Proceedings of the 32nd DAAAM International Symposium, pp.0593-0599, B. Katalinic (Ed.), Published by DAAAM International, ISBN 978-3-90273433-4, ISSN 1726-9679, Vienna, Austria

DOI: $10.2507 / 32$ nd.daaam.proceedings.084

\begin{abstract}
Experimental studies have been carried out on high-voltage overhead power lines (HVOPL) in order to determine the energy characteristics of the induced energy in the ground wire cable, taking into account the peculiarities of its grounding. The experimentally obtained results have been used in the development of simulation models of the HVOPL-500 sections located between the anchor supports. Simulation models of the anchor span with different numbers of supports have been built. As a result of simulation experiments, the dependences of power consumption as a function of the load resistance and voltage across the ground wire have been obtained. On the basis of the obtained dependences, recommendations have been developed for various options of power supply for the telemetry system components placed on the HVOPL-500 kV supports. In the course of simulation experiments, there have been determined the maximum values of power and the corresponding voltage values on the ground wire as a function of the load resistance value. Technical solutions and recommendations have been proposed for providing power supply to the components of diagnostic systems and monitoring the state of structural elements of the supports.
\end{abstract}

Keywords: ground wire; high-voltage overhead power line; induced energy; power supply of diagnostic and monitoring systems.

\section{Introduction}

High-voltage overhead power lines (HVOPL) are the main facilities for transporting electricity from producers to consumers. In Kazakhstan, where the length of the HVOPL with a voltage of 110-500 kV is more than 70 thousand km, reducing losses in the course of electricity transportation is a particularly urgent scientific and technical task [1]. In world practice, research and development on remote monitoring systems designed for monitoring and diagnostics of HVOPL are known [2], [3]. The known systems of remote HVOPL monitoring contain a measuring unit, telemetric communication channels and power supply elements [4]-[7]. Due to the remarkable remoteness of the HVOPL from the settlements, there is a problem with organizing the power supply to the components of the remote monitoring system placed on the supports. Power consumption of electrical equipment located on the support does not exceed $50 \mathrm{~W}$ at a voltage of $12 \mathrm{~V}$. 
At present, the following energy sources can be used to power the monitoring system units: solar batteries, converters the energy of the electromagnetic field from the HVOPL conductive wires [5]. Solar batteries (SB) with additionally mounted accumulator batteries $(\mathrm{AB})$ are the most common option in world practice. The CAT-1, DiLin, EMO, ThermalRate System, Snow Load Monitoring Systems operate on solar batteries that are directly mounted on the HVOPL supports [8]. They are used in lines ranging from 110 to $735 \mathrm{kV}$. However, with power supply from solar panels, a number of problems arise that reduce the reliability of the SB operation [9]. Due to the uneven SB operation (especially on cloudy days), the algorithm of the monitoring system is required to minimize power consumption. Implementation of a power supply from an SB with an additional $\mathrm{AB}$ requires high costs since electronic units must be placed on a large number of supports.

The power supply can also be performed from electromagnetic fields of the HVOPL conductive wires [6]. Works [4][5] describe low-energy options of the monitoring system power supply. The energy of the considered types of sources is not sufficient to power the local systems and indicator sensors located on the support. There is another unconventional method of generating energy for the diagnostic system that is based on the use of induced energy in-ground wire cables. The idea of obtaining induced energy from a ground wire was proposed by the Canadian Hydro-Québec Research Institute, but it was not brought to implementation and remained unrealized [10]. This work, there are analysed the possibilities of using this method of power supply to the blocks of the distributed protection system and diagnostics of structural elements of supports for high-voltage power lines of $220-500 \mathrm{kV}$ [6]-[7].

\section{Research methods}

In this work, experimental studies have been carried out at the HVOPL-220 kV in order to determine the energy characteristics of the induced energy in the ground wire cable, taking into account the peculiarities of its grounding. At this, the method of simulation experiments has been used. The data obtained in the process of experimental studies, in accordance with the principle of similarity, have been used in the development of simulation models and in carrying out simulation experiments for the HVOPL-500 kV. Simulation experiments consisted of three stages: 1. Obtaining the dependences of power consumption as a function of active load resistance for different numbers of supports. 2. Determining the ground wire voltage dependences as a function of the value of the load resistance for different numbers of supports. 3. Determining a sufficient number of intermediate spans to provide power supply to the diagnostic system components located on the supports.

\section{Characteristics of the object and its experimental studies}

The objects of research are conductive elements of structures and ground wires of HVOPL-220 kV and HVOPL-500 $\mathrm{kV}$. There have been studied the energy characteristics of the energy induced in the ground wire of a high-voltage power transmission line. The design of the ground wire for HVOPL-220 and HVOPL-500 is identical. As a rule, ground wire on supports is suspended on insulators and grounded at one end of anchor support [5]. In order to assess the energy characteristics, scientists of Karaganda Technical University, jointly with specialists of the KEGOC JSC, have carried out experimental studies of the induced electricity technical characteristics. The open-circuit voltage and short circuit current have been measured in partially de-energized conductive wires on the $220 \mathrm{kV}$ power transmission line. During the measurements, it has been found that the average voltage across the ground wire did not exceed $100 \mathrm{~V}$ at short-circuit current lower than $2 \mathrm{~A}$. The induced energy in the ground wire is sufficient to power the electronic diagnostic units located on the support. In the presence of electricity in the conductive wires, the induced energy in the ground wire is present regardless of climatic conditions and time of day.

The results obtained experimentally have been used in the development of simulation models of the HVOPL-500 sections located between the anchor supports. Technical characteristics of the induced energy substantially depend on the anchor span length. In the HVOPL-500 sections in the Karaganda region, the length of the anchor span varies from 2.5 $\mathrm{km}$ to $17.5 \mathrm{~km}$. Each anchor span contains from 8 to 58 supports. In this work, the energy characteristics of the induced energy have been studied, taking into account the length of the inter-anchor spans under the conditions of the normal operation of the HVOPL without taking into account the effect of lightning discharges, atmospheric phenomena (ice formations) and switching overvoltage in conductive wires [11]-[12].

\section{Developing a simulation model}

A simulation model of the induced voltage distribution in the ground wire cable along its entire length of one anchor span has been developed in the MATLAB-Simulink software environment. The model considers sections of the anchor span of HVOPL-500 kV with the initial data presented in Table 1. The static characteristics of the energy induced in the ground wire have been studied in the normal mode of operation, without taking into account the disturbing effects (disturbing factors) on the characteristics of the induced energy. The equivalent circuit of the support is shown in Fig. 1. The equivalent circuit of the support includes the distribution of the capacitive components, taking into account the design features within one portal-type support. Ground wire cables 2 and 4 are suspended on suspension insulators 1 and 3 , respectively. Conductive wires 6,8 and 10 suspended on suspension insulators 5, 7 and 10, respectively, have a capacitive connection with ground wire cables. 
Moreover, each ground wire cable is geometrically located at the same distance from the two phases of the conductive wires. Ground wire cable 2 has a capacitive connection $(\mathrm{C} 1$ and $\mathrm{C} 2)$ with conductive wires 6 and 8 , and ground wire cable 4 has a capacitive connection (C3 and C4) with conductive wires 8 and 10. The value of the capacitance between the conductive wires and the ground wire cable is determined by the dielectric constant of the air, the diameter of the cables and their length along the intermediate span. Additionally, the live wires have capacitance to the ground (C5, C6 and C7).

\begin{tabular}{|c|c|}
\hline 1. The line section length & $264 \mathrm{KM}$ \\
\hline 2. The conductive wire brand & AC-300/39, AC-300/66 \\
\hline 3. The wire section diameter & $25 \mathrm{~mm}$ \\
\hline 4. The ground wire brand & $2 \times \mathrm{TK}-11$ \\
\hline 5. The diameter of the ground wire section & $11 \mathrm{~mm}$ \\
\hline 6. The number of chains on the support & one \\
\hline 7. The number of wires per phase & three \\
\hline \multicolumn{2}{|l|}{ 8. The anchor span length: } \\
\hline a) $\quad$ minimum & $2500 \mathrm{~m}$ (8 supports) \\
\hline b) maximum & $17500 \mathrm{~m}$ (58 supports) \\
\hline
\end{tabular}

Table 1. Technical Specifications of the HVOPL Ek-GRES-PS Nura Section

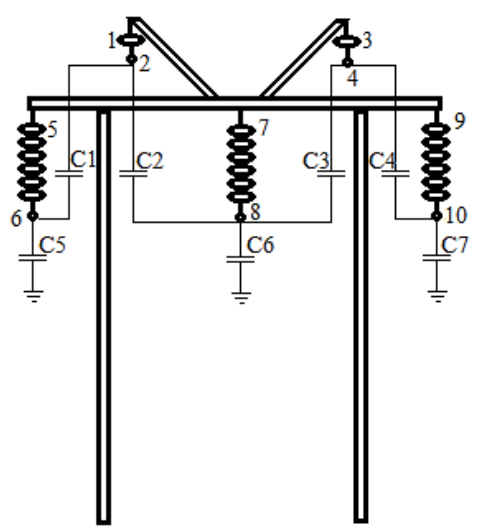

Fig. 1. The equivalent support circuit

The distance between live wires and ground is several times greater than the distance between the live wire and the ground wire. The number of intermediate spans within the anchor span in the analyzed section of HVOPL-500 varies in the range from 8 to 58, which corresponds to the number of supports at the minimum and maximum distances, respectively, within the anchor span. The equivalent circuit of an anchor span section with a ground wire cable is a sequential chain of span models describing the processes in the ground wire cable between the supports (Fig. 2).

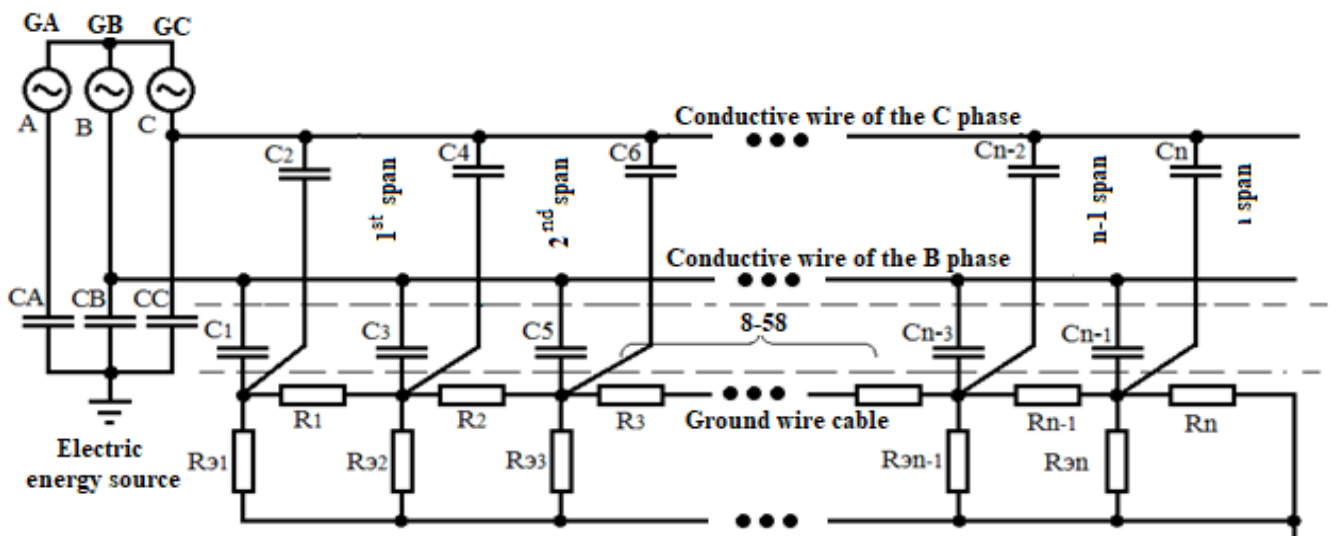

Fig. 2. The equivalent circuit of an anchor span section with a ground wire cable

One end of the ground wire cable is grounded on the anchor support, and the opposite end is structurally fixed to the suspended insulator. In the equivalent circuit, the electric capacitance between the supports relative to phase $C$ of the conductive wire is modelled by capacitors $\mathrm{C} 2, \mathrm{C} 4, \mathrm{C} 6, \mathrm{Cn}-2$ and $\mathrm{Cn}$, and between the conductive wire of phase $\mathrm{B}$ and the ground wire it is modelled by capacitors $\mathrm{C} 1, \mathrm{C} 3, \mathrm{C} 5, \mathrm{Cn}-3$ and $\mathrm{Cn}-1$. 
The active resistance of the ground wire between the supports is modelled by resistors R1, R2, R3, Rn-1 and Rn and the load characterizing the energy consumption of the local system for collecting, processing and transmitting the information of the state of structural elements to the control room is modelled by resistors Rэ1, Rэ2, Rэ3, Rэn-1 and Rэn. The numerical values of the parameters of the equivalent circuit elements are presented in Table 2.

\begin{tabular}{|c|c|c|c|}
\hline Circuit elements & $\mathrm{CA}, \mathrm{CB}$ and $\mathrm{CC}, \mathrm{pF}$ & $\mathrm{C} 1 \div \mathrm{Cn}, \mathrm{pF}$ & $\mathrm{R} 1 \div \mathrm{Rn}, \mathrm{Ohm}$ \\
\hline Value & $2.2710^{-6}$ & $1.310^{-9}$ & 0.43 \\
\hline
\end{tabular}

Table 2. The Parameters of the Equivalent Circuit Elements

The simulation model for the equivalent circuit of the anchor span is shown in Fig. 3.

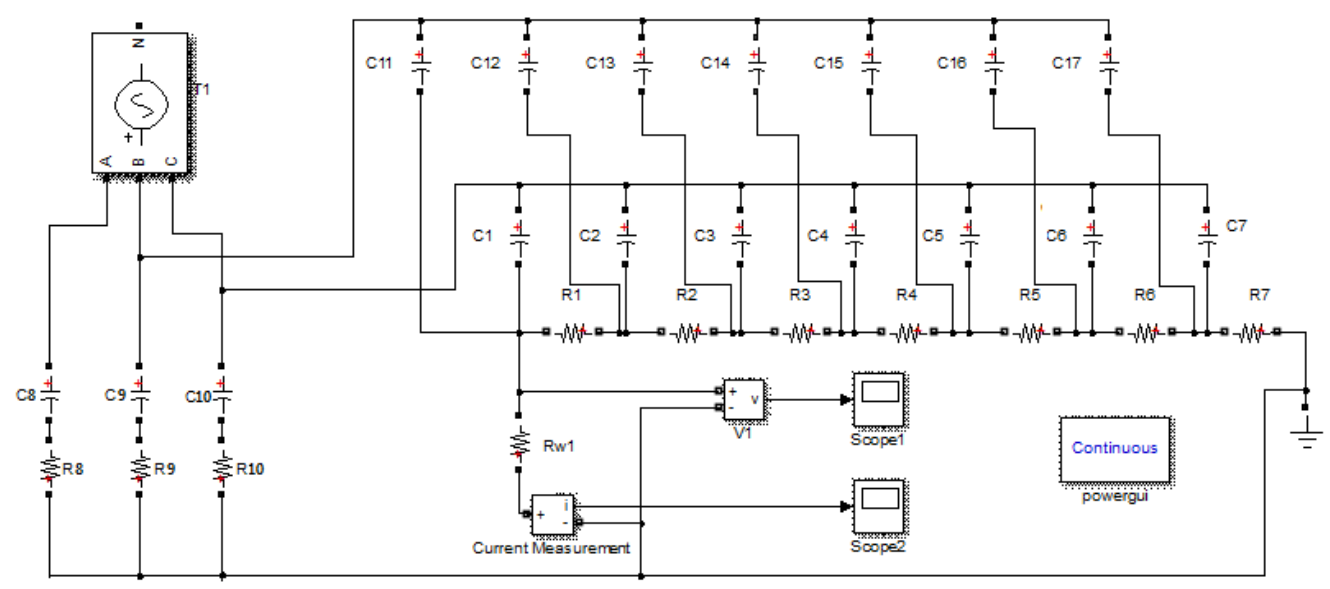

Fig. 3. The simulation model of the anchor span with seven supports

Simulation experiments have been carried out for two extreme values corresponding to the minimum (8) and maximum (58) number of supports for one anchor span. A feature of the simulation model is the need to include currentlimiting resistors R8, R9 and R10 in series with capacitors C8, C9 and C10, in order to prevent infinitely large current at the moment of the simulation experiment start. The values of these resistances are selected from the condition of obtaining no more than $1 \%$ error of simulation experiments. At the same time, the load RW1 simulates power consumption of the components of the remote diagnostics system located on the supports.

\section{Discussing the results of the studies}

As a result of simulation experiments, the dependences of power consumption as a function of the load resistance and voltage across the ground wire as a function of the load resistance have been obtained. The dependences have been obtained for 8, 16, 24 and 32 supports. On the basis of the dependences obtained, recommendations have been developed for various options of power supply to the system components placed on the HVOPL-500 kV supports. As a result of implementing the first stage, the dependences of power consumption as a function of the load resistance have been obtained. The dependencies are shown in Fig. 4. The Figure shows that the dependences of power consumption as a function of the load resistance are of an extreme nature. Simulation experiments have been carried out for different numbers of supports in the anchor span.

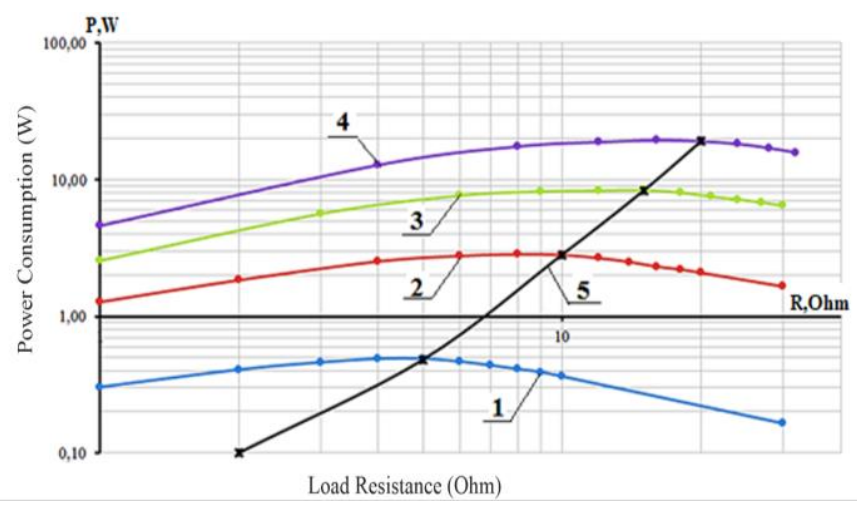

Fig. 4. The dependences of power consumption as a function of the load resistance 
In the process of simulation experiments, the number of supports varied in multiples of 8 . In Fig. 4, they are numbered counting from the grounded end of the lightning storm on the anchor support: - for the first eight supports - dependence 1 ; - for the first 16 supports - dependence 2; - for the first 24 supports - dependence 3; - for the first 32 supports dependence 4 . Dependence 5 is plotted graphically by the points of extreme values of dependences 1-4. The maximum power of the energy induced in the ground wire in the section of the first eight supports is $0.5 \mathrm{~W}$ with a load resistance of $5 \mathrm{Ohm}$. The power consumed by the elements of the system changes in different modes of their operation. For this reason, the power of the energy induced in the ground wire can be insufficient to power the system components.

The internal resistance of the power source is mainly determined by the resistance of the ground wire. As the length of the ground wire increases, its resistance increases. The extremum is reached when the internal resistance of the power source and the load resistance is equal. In this regard, with increasing the internal resistance of the power source, the extremum shifts towards increasing the load resistance. An insignificant effect on the law of variation of the extremum as a function of load resistance (dependence 5 in Fig 4) is exerted by increasing the distributed electrical capacitance between the conductive wires of phases $\mathrm{B}$ and $\mathrm{C}$ and the ground wire with increasing the span between the anchor supports (Fig. 3), which determines nonlinearity of dependence 5 (Fig. 4). Thus, dependence 5 corresponds to the maximum value of the power supplied to the load as a function of the load resistance value for different numbers of supports in the anchor span. The optimal power values for the corresponding number of supports are presented in Table 3.

\begin{tabular}{|l|c|c|c|c|}
\hline \multicolumn{1}{|c|}{ Diagram number (Figure 4) } & 1 & 2 & 3 & 4 \\
\hline Number of supports in the anchor span, pcs. & 8 & 16 & 24 & 32 \\
\hline Load resistance, Ohm & 3 & 6 & 9 & 12 \\
\hline Power supplied to load, W & 0.5 & 2.4 & 7.4 & 17.6 \\
\hline Load voltage, V & 1.52 & 5.2 & 11 & 19 \\
\hline
\end{tabular}

Table 3. The Optimal Power Values

An important parameter in matching the induced energy in the ground wire with the load parameters is load voltage for different numbers of supports in the anchor span. Due to the fact that the magnitude of voltage depends on the magnitude of the load resistance and the length of the anchor span, it is necessary to study the effect of the above parameters on the magnitude of the voltage across the ground wire at the point of load connection using simulation tools. In order to determine the magnitude of the amplitude value of voltage, dependencies are obtained as a function of the load resistance. The dependencies are shown in Fig. 5.

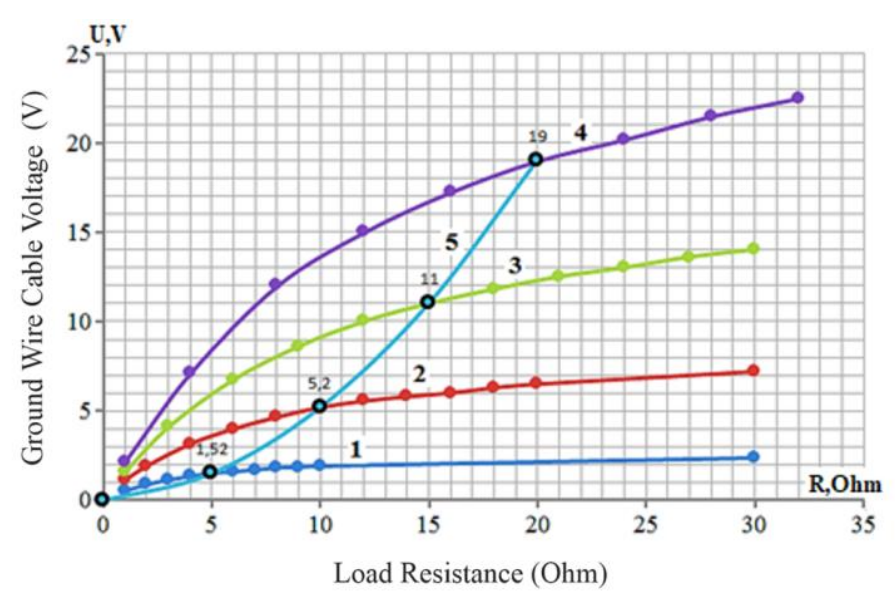

Fig. 5. The ground wire cable voltage dependences as a function of the load resistance

With increasing the load resistance in the range of $1 \div 30 \mathrm{Ohm}$, there is observed voltage increasing. With increasing the number of supports in the anchor span in the range of $8 \div 32$ pcs., there is also observed increasing voltage across the load in the range of $0.5 \div 22 \mathrm{~V}$. Fig. 5 shows dependence 5 of the voltage value for the optimal values of the load resistance. As a result of simulation experiments, it has been found that voltage across the load at the optimal value of the load resistance, with increasing the number of supports in the anchor span in the range of $8 \div 32$ pcs., increases according to the law that is close to the parabolic law in the range of $1.5 \div 19 \mathrm{~V}$. The voltage value on the ground wire for different numbers of supports and the optimal value of the load resistance is presented in Table 3. Thus, with increasing the number of supports more than 24 pcs., starting with the anchor support, where the grounding of the ground wire cable is implemented, the power of the energy induced in the ground wire can be considered sufficient to power the local system. With the number of supports from 16 to 24 , it is desirable to use buffer energy storage in the form of a storage battery or high-capacity capacitors when developing a power supply unit. 
In the section of the anchor span, where there are fewer than 16 intermediate spans of the induced energy in the ground wire, the power of the energy induced in the ground wire is not sufficient to power the elements of local systems. Therefore, it is necessary to use other technical solutions, for example, solar batteries with buffer energy storage.

\section{Conclusion}

The unconventional method of generating power energy for the diagnostic and monitoring systems of high-voltage overhead power lines, based on the use of induced energy in-ground wire cables, taking into account the peculiarities of its grounding, was not brought to implementation and required study. The paper analysed the possibilities of using this power supply method to the diagnostic systems for high-voltage power lines of $220-500 \mathrm{kV}$. The equivalent circuit of the anchor span section with a ground wire cable for the HVOPL-500 kV "HVOPL Ek-GRES-PS Nura" section has been developed. A simulation model of the distribution of the induced energy in the lightning protection wire of the anchor span has been developed using the MATLAB software package. In simulation experiments, the maximum power values and the corresponding voltage values across the ground wire have been determined as a function of the load resistance value. The possibilities of power supply to the components of diagnostic and monitoring systems located on the HVOPL supports have been determined. There have been proposed technical solutions and recommendations for providing power supply to the components of diagnostic systems and monitoring the state of structural elements of the supports. The results obtained can be extended to HVOPL-500 kV with portal type supports and a similar structure for placing ground wires on the supports. In the future, the power supply system requires development, where the use of solar panels with buffer energy storage, when the induced energy in the ground wire is not sufficient to power the elements of local systems.

\section{Acknowledgements}

This work has been supported by grant funding for scientific and (or) scientific-technical projects at the level of the Ministry of Education and Science of the Republic of Kazakhstan on topic No. IRN AR08856344 "Development of a complex for remote monitoring the state of structural elements of high-voltage overhead power lines during operation based on intelligent noise-immune telemetry systems".

\section{References}

[1] Atakhanova, Z. \& Howie, P. (2007). “Electricity demand in Kazakhstan”, Energy Policy, Vol. 35, No. 7, 2007, pp. 3729-3743, DOI 10.1016/j.enpol.2007.01.005.

[2] Yang, Y., Xie, G., Xu, X. \& Jiang, Y. (2011). “A Monitoring System Design in Transmission Lines based on Wireless Sensor Networks", Energy Procedia, Vol. 12, 2011, pp. 192-199, ISSN 1876-6102, DOI 10.1016/j.egypro.2011.10.027.

[3] Morozovska, K. \& Hilber, P. (2017). "Study of the Monitoring Systems for Dynamic Line Rating", Energy Procedia, Vol. 105, 2017, pp. 2557-2562, ISSN 1876-6102, DOI 10.1016/j.egypro.2017.03.735.

[4] Peabody, A. (2009). "Evolution of Real-time Monitoring and its Future Benefits", IWAIS, No. XIII, Sep. 2009.

[5] Kaverin, V. V.; Khomchenko, V. G. \& Yezhebayeva, Sh. Ye. (2020, Jun.). Review and analysis of existing power supplies for monitoring systems located on the supports of overhead power lines, Presented at Proceedings (Saginov's Readings). No. 12, pp. 743-745.

[6] Breido, I., Kaverin, V. \& Ivanov V. (2018). Telemetric Monitoring Insulation Condition of High Voltage Overhead Power Lines, Presented at Proceedings of the 29th DAAAM International Symposium, pp. 0319-0328, B. Katalinic (Ed.). DOI 10.2507/29th.daaam.proceedings.046.

[7] Breydo, I., Kaverin, V., Ivanov, V., Voytkevich, S. \& Levin, I. (2017). "Distributed system of protection and diagnostics of support structural elements of high-voltage power lines", EAI Endorsed Transactions on Energy Web. 4. 152983. 10.4108/eai.3-8-2017.152983. 017.

[8] Uski-Joutsenvuo, S. \& Pasonen, R. (2013, Feb.). Maximizing power line transmission capability by employing dynamic line ratings - technical survey and applicability in Finland, VTT Technical Research Centre of Finland, Finland.

[9] Bessel, V. V.; Kucherov, V. G. \& Mingaleyeva R. D. (2016). Studying solar photovoltaic cells. (Publishing Center of the Russian State University of Oil and Gas named after I.M. Gubkin).

[10] Blais R. \& Berthiaume R. (1980). "Supplying fixed and stroboscopic light beacons from the overhead ground wire on $735 \mathrm{kV}$ transmission lines", IEEE Transactions on Power Apparatus and Systems, Vol. PAS-99, No. 1 Jan./Feb, 1980.

[11] Breido, I. V.; Kaverin, V. V.; \& Voitkevich, S. V. The system of cathodic protection of elements of supports of high-voltage power lines sections, RK Patent No. 29977, May 19, 2015.

[12] Breido, I. V.; Kaverin, V. V.; Em, G. A.; Sichkarenko, A. V.; Ivanov, V. A. \& Voitkevich, S. V. (2018). Method of telemetric measurement of ice loads of high-voltage power lines and a system for its implementation, RK Patent No. 33249, Oct. 26, 2018. 